\title{
Very Heavy Flavored Dibaryons
}

\author{
Jean-Marc Richard $\odot,,^{1, *}$ Alfredo Valcarce, ${ }^{2, \dagger}$ and Javier Vijande ${ }^{3, \$}$ \\ ${ }^{1}$ Université de Lyon, Institut de Physique des 2 Infinis de Lyon, IN2P3-CNRS-UCBL, \\ 4 rue Enrico Fermi, 69622 Villeurbanne, France \\ ${ }^{2}$ Departamento de Física Fundamental, Universidad de Salamanca, 37008 Salamanca, Spain \\ ${ }^{3}$ Unidad Mixta de Investigación en Radiofisica e Instrumentación Nuclear en Medicina (IRIMED), \\ Instituto de Investigación Sanitaria La Fe (IIS-La Fe)-Universitat de Valencia (UV) and IFIC (UV-CSIC), \\ 46100 Valencia, Spain
}

(Received 5 February 2020; accepted 13 May 2020; published 27 May 2020)

\begin{abstract}
We explore the possibility of very heavy dibaryons with three charm quarks and three beauty quarks, $b b b c c c$, using a constituent model which should lead to the correct solution in the limit of hadrons made of heavy quarks. The six-body problem is treated rigorously, in particular taking into account the orbital, color, and spin mixed-symmetry components of the wave function. Unlike a recent claim based on lattice QCD, no bound state is found below the lowest dissociation threshold.
\end{abstract}

DOI: 10.1103/PhysRevLett.124.212001

Introduction.-Apart from the atomic nuclei, there is no evidence, so far, for stable multiquark states in the hadron spectrum. At best, there are very interesting resonances which lie above their lowest dissociation threshold. Sometimes the corresponding fall-apart decay is suppressed, and a rather narrow resonance is observed. There are several recent reviews, see, for instance Refs. [1-8].

Several stable multiquarks have been predicted along the years, based on various mechanisms. The famous $H$ (uuddss) by Jaffe [9] tentatively gets its binding from coherences in the chromomagnetic sector. The same mechanism is also at work in other configurations, such as the anticharmed pentaquark [10-12]. But, when a full quark model calculation is performed the stability does not survive the breaking of flavor $\mathrm{SU}(3)$ [13,14], nor the unavoidable dilution of the structure that weakens the strength of the chromomagnetic terms $[15,16]$.

Another possibility is the combination of two heavy quarks and two light antiquarks, $Q Q \bar{q} \bar{q}$. Here, there is a favorable chromomagnetic effect if $\bar{q} \bar{q}=\bar{u} \bar{d}$, but the novelty is the chromoelectric binding that exploits the breaking of charge conjugation when the quark-toantiquark mass ratio $M / m$ departs from unity. This is the same mechanism that makes the hydrogen molecule much more stable than the positronium molecule [4].

In recent years, lattice QCD based studies have managed to reach calculations at almost the physical pion mass.

Published by the American Physical Society under the terms of the Creative Commons Attribution 4.0 International license. Further distribution of this work must maintain attribution to the author(s) and the published article's title, journal citation, and DOI. Funded by SCOAP .
Thus, the HAL QCD Collaboration has studied dibaryons containing light or strange quarks establishing the existence of barely bound $\Omega \Omega$ [17] and $N \Omega$ [18] states close to the unitary limit. Lattice calculations with heavy quarks are advantageous over the light counterparts because the two point correlators are less noisy and the signal to noise is far better.

Recently, Junnarkar and Mathur [19] reported the first lattice QCD study of dibaryons with heavy quark flavors. They evaluated deuteronlike dibaryon structures with the quark contents $(u u c)(u c c),(s s c)(s c c),(u u b)(u b b)$, $(s s b)(s b b)$, and $(c c b)(c b b)$ and quantum numbers $(I) J^{P}=(0) 1^{+}$. The authors conclude that $(s s c)(s c c)$, $(s s b)(s b b)$, and $(c c b)(c b b)$ are clearly below the twobaryon thresholds. It is important to note that for these systems the lowest two-baryon threshold is always made of two spin $3 / 2$ baryons with quark content $(Q Q Q)(q q q)$. The existence of such deep bound states in the heavy quark sector should be captured in any model having the right QCD properties in the heavy quark sector. In particular a constituent model approach should reflect such deep binding, if it exists [20,21].

Thus, the aim of the present Letter is to revisit the aforementioned configurations in a full-fledged calculation considering the internal mixed symmetry components of the hexaquark wave function, and study whether or not three $c$ quarks and three $b$ quarks do form a bound state.

Model and method.-We have calculated the energy of bbbccc and its threshold using a standard constituent model. The main assumption, on which we shall come back in the last section, consists of adopting a pairwise interaction with a color-octet exchange structure. The interaction has two terms only, i.e., spin-orbit and tensor 
forces are neglected: a spin-independent or chromoelectric interaction that reads

$$
V_{c}=-\frac{3}{16} \sum_{i<j}\left(\tilde{\lambda}_{i} \cdot \tilde{\lambda}_{j}\right)\left(-a / r_{i j}+b r_{i j}\right)
$$

and a spin-spin or chromomagnetic interaction given by

$$
V_{s}=-\frac{3}{16} \sum_{i<j}\left(\tilde{\lambda}_{i} \cdot \tilde{\lambda}_{j}\right)\left(\vec{\sigma}_{i} \cdot \vec{\sigma}_{j}\right) \frac{a_{s s}}{m_{i} m_{j}}\left(\frac{\mu}{\pi}\right)^{3 / 2} \exp \left(-\mu r_{i j}^{2}\right),
$$

with $a=0.2 / \hbar c, b=0.4 \hbar c, a_{s s}=2.0, \mu=1.0 /(\hbar c)^{2}$, $m_{c}=1.3 \mathrm{GeV}$, and $m_{b}=4.66 \mathrm{GeV}$.

In such potential, the ground state of three quarks corresponds to a stable baryon. A constant term can be introduced in Eq. (1), $-(3 / 16) C\left(\tilde{\lambda}_{i} \cdot \tilde{\lambda}_{j}\right)$, but it would shift each baryon of the threshold by $C$ and every dibaryon by $2 C$ and thus cannot modify the conclusions about the stability or instability of the latter.

The above potential corresponds to a fit by Semay and Silvestre-Brac [22]. It is worth emphasizing that the parameters are constrained in a simultaneous fit of 36 wellestablished meson states and 53 baryons, with a remarkable agreement with data, as can be seen in Table 2 of Ref. [22]. Notwithstanding, it has been checked that the conclusions dealing with stability or instability of multiquarks survive sizable variations of the parameters, and changes in the functional form adopted for the potential.

The color, spin, and orbital structure of the wave function has been firstly worked out in a basis (123)-(456) = $(b b b)-(c c c)$, where the Pauli principle is imposed in a more apparent manner. The color of each cluster is either the antisymmetric singlet 1 or the pair of mixed-symmetry octet $\left\{8_{\lambda}, 8_{\rho}\right\}$, which are symmetric or antisymmetric under $1 \leftrightarrow 2$ (or $4 \leftrightarrow 5$ ), respectively. The color decuplet states do not contribute. As for the spin, we have either $S_{123}\left(\right.$ or $\left.S_{456}\right)=3 / 2$ or $1 / 2$, which are symmetric or mixed symmetry, respectively. Subsequently, the clustering $(b b c)-(c c b)$ has also been used for checking purposes. In this case, the wave function has been obtained by the transformation of the original properly antisymmetrized wave function in the (123)-(456) $=(b b b)-(c c c)$ basis. The spin-color algebra has also to be transformed into the new coupling. Let us note that, in contrast to a deuteronlike dibaryon, the color-spin-radial wave function must be antisymmetric due to the nonexistence of a flavor-antisymmetric component.

In the ground state of the six-quark system, the orbital wave function is dominated by the components that are symmetric in both $b b b$ and $c c c$ sets of permutations, but mixed-symmetric orbital wave functions are also included. The recoupling of the spins is obvious. The Clebsch-Gordan coefficients of the color recoupling to an overall singlet are taken from Ref. [23]. The coupling of two or three mixedsymmetry components to an overall symmetric or antisymmetric state is explained, e.g., in Ref. [24]. The variational wave functions are based on Gaussians of the type

$$
\exp (-\varphi / 2), \quad \varphi=\sum_{1 \leq i<j \leq 6} a_{i j} r_{i j}^{2}
$$

and appropriate combinations of their permutations [24]. In practice, the interparticle distances $\vec{r}_{i j}$ are expressed in terms of standard Jacobi coordinates which diagonalize the intrinsic kinetic energy. Such a properly symmetrized combination of permutations also leads to nondiagonal Jacobi coordinate products, i.e., $\vec{x}_{i} \cdot \vec{x}_{j}$, that generate nonzero internal orbital angular momenta in the wave function.

It is safer to proceed by steps. For each state of total spin, from $S=3$ to $S=0$, we first considered symmetric orbital functions associated to color-singlet clusters, afterwards the admixture of color octet components coupled to mixedsymmetry spin states was included. In a third stage, the corrections due to mixed-symmetry orbital components were analyzed. In principle, especially for $S=1$ or $S=0$, the number of coupled components can become large, and lead to rather delicate numerical calculations. In such a case, it is wise to introduce the corrections one by one, and add up the corresponding energy shifts. A similar strategy is used, e.g., when treating the high partial waves of the hyperspherical expansion [24].

Results.-We first calculated the energy of the various baryons $b^{n} c^{3-n}$, and found without surprise that the lowest threshold is made of $(b b b)+(c c c)$. The convexity of the baryon spectrum when the masses are varied is reviewed, e.g., in Refs. [24-27]. With the parameters of the model of Ref. [22] the lowest threshold $\Omega_{b b b}\left(3 / 2^{+}\right)+\Omega_{c c c}\left(3 / 2^{+}\right)$ has a mass of $19.082 \mathrm{GeV}$. The details are given in Table I.

For the hexaquarks bbbccc, the energy was always found above the threshold, even when color octet and/or mixed-symmetry orbital and spin wave functions are introduced for both three-quark clusters. More precisely as follows:

Spin 3: As the spin wave function is fully symmetric, we have targeted an additional antisymmetric wave function made of mixed-symmetry orbital and color components.

Spin 2: Besides the wave function mentioned above we included antisymmetric wave functions made of

TABLE I. Masses, in GeV, of baryons within the constituent model of Ref. [22].

\begin{tabular}{lcc}
\hline \hline Baryon & $S=3 / 2$ & $S=1 / 2$ \\
\hline$b b b$ & 14.253 & $\ldots$ \\
$c c c$ & 4.829 & $\ldots$ \\
$b b c$ & 11.162 & 11.137 \\
$c c b$ & 8.023 & 7.972 \\
\hline \hline
\end{tabular}


mixed-symmetry orbital, color, and spin components for one of the subclusters of three identical quarks. Such a component resembles two baryons with $S=1 / 2$ and $S=3 / 2$.

Spin 0 and 1: Besides the components mentioned above we included antisymmetric wave functions made of mixedsymmetry orbital, color, and spin components for both subclusters of three identical quarks. Such a component would take account of internal states made of two $S=1 / 2$ baryons.

The negligible contribution coming from mixed symmetry components of the wave function can be easily understood by calculating their energy if considered separately. Thus, the $S=3$ state containing mixed symmetry color and orbital components has an energy of $19.869 \mathrm{GeV}$, to be compared to the $19.098 \mathrm{GeV}$ of the color-orbital antisymmetric-symmetric component. Similarly, the $S=1$ state containing mixed symmetry orbital-color-spin components has an energy of $19.838 \mathrm{GeV}$, to be compared to the $19.098 \mathrm{GeV}$ of the lowest component. They are far enough to have a rather weak coupling with the dominant state. Moreover, the adjustment of the parameters always led to very small and even vanishing values for the $a_{i j}$ coefficients with $i \in\{1,2,3\}$ and $j \in\{4,5,6\}$. This is a clear signature of a converged variational calculation in the absence of bound states.

Note that in the case of equal masses $m_{b}=m_{c}$, there is no bound state either, and this can be understood by an argument of symmetry breaking that was already developed for comparing $\overline{3} 3$ and $6 \overline{6}$ tetraquarks with equal masses [28]. Let, indeed,

$$
H=\sum_{i=1}^{N} \frac{\vec{p}_{i}^{2}}{2 m}+\sum_{1 \leq i<j \leq N} g_{i j} V\left(r_{i j}\right),
$$

be an Hamiltonian with attractive pair potential $V$, and the cumulated strength fixed, say $\sum g_{i j}=N /(N-1) / 2$. The energy is maximal for equal strengths $g_{i j}=1$ and is a concave function of these variables $g_{i j}$, as they enter $H$ linearly. So, schematically, the more asymmetric the $\left\{g_{i j}\right\}$ distribution, the lower the energy. Clearly, for $N=6$, one can hardly find a distribution more asymmetric than that of the threshold with $g_{12}=g_{23}=g_{13}=g_{45}=g_{56}=g_{46} \neq 0$ and the other $g_{i j}=0$, and in the regime of heavy quarks such as $b$ and $c$, the hyperfine corrections play a minor role and cannot generate binding by themselves. Our numerical calculations also show that the results based on a frozen set of color coefficients are not significantly modified by the coupling of the various allowed channels.

Discussion and outlook.-The main conclusion of our accurate six-body calculation is the absence of binding for $b b b c c c$ and similar configurations. This outcome is based on an explicit potential model and several variants of this potential. The principal reason for this instability is the constraint of antisymmetrization in both the $b$ and the $c$ sectors, which prohibits the mixing of all possible configurations corresponding to a color-neutral hexaquark of given spin. As a toy model, we calculated a configuration $b b^{\prime} b^{\prime \prime} c c^{\prime} c^{\prime \prime}$ with the same masses as before and the same potential, Eqs. (1) and (2), but nonidentical $b$-type and $c$-type of quarks. Then a binding of about $100 \mathrm{MeV}$ is obtained.

A related investigation consists of questioning the prescription

$$
V=-\frac{3}{16} \sum_{i<j} \tilde{\lambda}_{i} \tilde{\lambda}_{j} v\left(r_{i j}\right)
$$

where the normalization is such that $v(r)$ is the quarkonium potential. For the linear part of the interaction, say $v(r)=\sigma r$, a challenging alternative consists of building the minimal string or set of strings linking the quarks. For a baryon, this leads to the well-known Fermat-Torricelli construction

$$
V=\sigma \min _{J}\left(r_{1 J}+r_{2 J}+r_{3 J}\right),
$$

which gives an energy slightly higher than the additive rule Eq. (5). However, for tetraquarks, if one generalizes Eq. (6) by a combination of flip-flop and connected strings, namely,

$$
\begin{aligned}
V & =\sigma \min _{J}\left(r_{3}-r_{1}+r_{4}-r_{2}, r_{3}-r_{2}+r_{4}-r_{1}, V_{Y Y}\right), \\
V_{Y Y} & =\min _{J K}\left(r_{1 J}+r_{2 J}+r_{J K}+r_{3 K}+r_{4 K}\right),
\end{aligned}
$$

then the potential becomes much more favorable as compared to the additive rule, and binding of $Q Q^{\prime} \bar{q} \bar{q}^{\prime}$ is obtained already for a mass ratio $M / m=1$. However, an important restriction has to be enforced: the quarks and the antiquarks should be different, i.e., not submitted to the Pauli principle, even so equal masses are adopted, for the sake of simplicity [29].

If the same strategy of a string confinement is adopted for six quarks, with the potential resulting from a minimization over various connected strings and flip-flop configurations, then binding is also obtained [30]. For a purely linear interaction $v(r)=r$, and a mass ratio $M / m=1$ associated with a light mass $m=1$, it was found that the hexaquark $Q Q Q q q q$ is bound by about 0.491 in such dimensionless units. If one restores the appropriate scales, namely $m_{c} \simeq 1.5 \mathrm{GeV}$ and a string tension $\sigma \simeq 0.2 \mathrm{GeV}^{2}$, this corresponds to about $0.1 \mathrm{GeV}$. But, again, the possibility of optimizing the strings continuously as the quarks move requires a full waiver of antisymmetrization [30]. In other words, a constituent model leaned on the string confinement predicts $b b^{\prime} b^{\prime \prime} c c^{\prime} c^{\prime \prime}$ to be stable with a binding energy of about $100 \mathrm{MeV}$. This state disappears when the $b$ and the $c$ become identical. 
To conclude, potential models, when treated seriously, do not lead to a proliferation of stable multiquarks. In particular, there is no evidence for any stable super-heavy hexaquark of the type bbbccc.

This work has been partially funded by Ministerio de Economía, Industria y Competitividad and EU FEDER under Contracts No. FPA2016-77177 and No. RED2018102572-T.

*j-m.richard@ipnl.in2p3.fr

†valcarce@usal.es

\#javier.vijande@uv.es

[1] R. A. Briceno et al., Issues and opportunities in exotic hadrons, Chin. Phys. C 40, 042001 (2016).

[2] H.-X. Chen, W. Chen, X. Liu, and S.-L. Zhu, The hiddencharm pentaquark and tetraquark states, Phys. Rep. 639, 1 (2016).

[3] A. Esposito, A. Pilloni, and A.D. Polosa, Multiquark resonances, Phys. Rep. 668, 1 (2017).

[4] J.-M. Richard, Exotic hadrons: Review and perspectives, Few Body Syst. 57, 1185 (2016).

[5] R. F. Lebed, R. E. Mitchell, and E. S. Swanson, Heavyquark QCD exotica, Prog. Part. Nucl. Phys. 93, 143 (2017).

[6] A. Ali, J. S. Lange, and S. Stone, Exotics: Heavy pentaquarks and tetraquarks, Prog. Part. Nucl. Phys. 97, 123 (2017).

[7] A. Ali, L. Maiani, and A. D. Polosa, Multiquark Hadrons (Cambridge University Press, Cambridge, 2019).

[8] N. Brambilla, S. Eidelman, C. Hanhart, A. Nefediev, C.-P. Shen, C. E. Thomas, A. Vairo, and C.-Z. Yuan, The $X Y Z$ states: Experimental and theoretical status and perspectives (2019).

[9] R. L. Jaffe, Perhaps a Stable Dihyperon, Phys. Rev. Lett. 38, 195 (1977); 38, 617(E) (1977).

[10] H. J. Lipkin, New possibilities for exotic hadrons: Anticharmed strange baryons, Phys. Lett. B 195, 484 (1987).

[11] C. Gignoux, B. Silvestre-Brac, and J. M. Richard, Possibility of stable multi-quark baryons, Phys. Lett. B 193, 323 (1987).

[12] J. Leandri and B. Silvestre-Brac, Systematics of $\bar{Q} q^{4}$ systems with a pure chromomagnetic interaction, Phys. Rev. D 40, 2340 (1989).

[13] J. L. Rosner, SU(3) breaking and the $H$ dibaryon, Phys. Rev. D 33, 2043 (1986).
[14] J.-M. Richard, A. Valcarce, and J. Vijande, Pentaquarks with anticharm or beauty revisited, Phys. Lett. B 790, 248 (2019).

[15] M. Oka, Flavor octet dibaryons in the quark model, Phys. Rev. D 38, 298 (1988).

[16] B. H. Kim et al. (Belle Collaboration), Search for an $H$-Dibaryon with Mass Near $2 m_{\Lambda}$ in $\Upsilon(1 S)$ and $\Upsilon(2 S)$ Decays, Phys. Rev. Lett. 110, 222002 (2013).

[17] S. Gongyo et al., Most Strange Dibaryon from Lattice QCD, Phys. Rev. Lett. 120, 212001 (2018).

[18] T. Iritani et al., $N \Omega$ dibaryon from lattice QCD near the physical point, Phys. Lett. B 792, 284 (2019).

[19] P. Junnarkar and N. Mathur, Deuteronlike Heavy Dibaryons from Lattice Quantum Chromodynamics, Phys. Rev. Lett. 123, 162003 (2019).

[20] M. Karliner and J. L. Rosner, Discovery of DoublyCharmed $\Xi_{c c}$ Baryon Implies a Stable $(b b \bar{u} \bar{d})$ Tetraquark, Phys. Rev. Lett. 119, 202001 (2017).

[21] E. J. Eichten and C. Quigg, Heavy-Quark Symmetry Implies Stable Heavy Tetraquark Mesons $Q_{i} Q_{j} \bar{q}_{k} \bar{q}_{l}$, Phys. Rev. Lett. 119, 202002 (2017).

[22] C. Semay and B. Silvestre-Brac, Diquonia and potential models, Z. Phys. C 61, 271 (1994).

[23] A. Alex, M. Kalus, A. Huckleberry, and J. von Delft, A numerical algorithm for the explicit calculation of SU(N) and SL(N,C) Clebsch-Gordan coefficients, J. Math. Phys. (N.Y.) 52, 023507 (2011).

[24] J.-M. Richard, The nonrelativistic three-body problem for baryons, Phys. Rep. 212, 1 (1992).

[25] A. Martin, J. M. Richard, and P. Taxil, About convexity properties of the baryon mass spectrum, Phys. Lett. B 176, 224 (1986).

[26] J. M. Richard and P. Taxil, Ground state baryons in the nonrelativistic quark model, Ann. Phys. (N.Y.) 150, 267 (1983).

[27] S. Nussinov and M. A. Lampert, QCD inequalities, Phys. Rep. 362, 193 (2002).

[28] J.-M. Richard, A. Valcarce, and J. Vijande, Few-body quark dynamics for doubly heavy baryons and tetraquarks, Phys. Rev. C 97, 035211 (2018).

[29] J. Vijande, A. Valcarce, and J.-M. Richard, Adiabaticity and color mixing in tetraquark spectroscopy, Phys. Rev. D 87, 034040 (2013).

[30] J. Vijande, A. Valcarce, and J. M. Richard, Stability of hexaquarks in the string limit of confinement, Phys. Rev. D 85, 014019 (2012). 